\title{
La relazione di cura e il benessere condiviso
}

\author{
Antonella Delle Fave ${ }^{1}$
}

\section{RIASSUNTO}

La relazione di cura può essere un importante opportunità di costruzione e condivisione di benessere, sia per il paziente che per l'operatore sanitario. Numerose ricerche negli ultimi due decenni hanno studiato questi aspetti, mettendone in evidenza le potenzialità e le ricadute positive per la qualità di vita dei lavoratori, dei loro assistiti, e della collettività in cui vivono.

Care relationship can represent an important opportunity to build and share well-being, both for patients and health professionals. In the last two decades, several studies have investigated these aspects, emphasizing their positive influence on the quality of life of workers, patients, and the communities they belong to.

\section{PAROLE CHIAVE}

Condivisione, promozione delle risorse, benessere lavorativo, eudaimonia

Sharing, resource promotion, well-being at work, eudaimonia

\section{LE PREMESSE CONCETTUALI}

Un importante criterio per definire la giusta distanza relazionale con il destinatario delle professioni di aiuto è quello di ottenere l'alleanza terapeutica, che scaturisce dalle caratteristiche emotivo/affettive della relazione con il paziente. Essa è promossa da tre aspetti dell'atteggiamento dell'operatore: empatia, coerenza, disponibilità ed apertura incondizionate. Più recentemente ne sono stati identificati e valorizzati anche aspetti cognitivi, quali la percezione da parte del paziente della rilevanza ed opportunità del trattamento proposto e la definizione condivisa degli obiettivi da raggiungere.

È utile, a tale proposito, chiarire cosa si intenda per empatia, trattandosi di un costrutto centrale nella relazione tra i professionisti della salute e i loro pazienti. Di questo termine sono state date varie definizioni, in base ai contesti specifici in cui il esso viene utilizzato. Quella più adeguata all'ambito della salute considera l'empatia una condizione di attivazione affettiva derivante dalla comprensione dello stato emotivo di un'altra persona, e che è congruente con esso.

L'empatia si manifesta in risposta ad emozioni altrui, sia positive che negative, ed implica la capacità dell'individuo di riconoscere e identificare le proprie reazioni emotive come risposte all'emozione dell'altra persona. L'empatia pertanto non conduce all'identificazione con il paziente e con il suo stato di sofferenza, bensì alla comprensione profonda (non solo razionale, ma anche cognitivo-affettiva) delle sue emozioni ed alla possibilità di

\footnotetext{
${ }^{1}$ Facoltà di medicina e Chirurgia, Università degli Studi di Milano
} 
mettere in atto comportamenti adeguati ad esse. Questa distinzione è di estrema importanza: il professionista, infatti, non deve far proprie le preoccupazioni, le ansie ed i dubbi del paziente, pena la perdita di oggettività e di appropriatezza nelle decisioni terapeutiche; egli deve invece riconoscerle e farsene carico nella gestione della relazione, allo scopo di accompagnare il paziente nel percorso decisionale e di gestione della malattia nel modo più corretto ed efficace.

Non bisogna peraltro dimenticare che ci troviamo in un contesto relazionale. II paziente come protagonista attivo della relazione può non essere sempre aperto alla costruzione di un rapporto di alleanza: sta quindi all'operatore facilitarne l'apertura, attraverso strumenti cognitivi e affettivi ai quali in questa sede possiamo solo accennare.

La prospettiva del paziente e quella dell'esperto devono completarsi reciprocamente, permettendo la costruzione di un percorso terapeutico condiviso, considerato adeguato dall'operatore sanitario e valutato consapevolmente e criticamente dal paziente. $\dot{E}$ di fondamentale importanza, a tale scopo, la condivisione di potere e responsabilità. Essa rimanda al coinvolgimento attivo del paziente nella decisione terapeutica, coinvolgimento che tuttavia è molto diverso dalla totale autonomia di scelta e che va supportato attraverso diverse azioni:

valorizzare l'esperienza del paziente (ovvero la sua conoscenza della malattia in prima persona) attraverso un ascolto accurato e l'apertura di finestre di opportunità che permettano al paziente di articolare in termini soggettivi la sua interpretazione dei sintomi, della loro evoluzione nel tempo e del loro significato;

spiegare al paziente con chiarezza e in termini comprensibili la sua situazione e le possibilità di intervento. È importante qui sottolineare la distinzione tra "informare" e "spiegare". L'informazione è neutra, standardizzata, caratterizzata dalla terminologia tecnica dell'ambito a cui si riferisce, e diretta a un interlocutore generico e aspecifico. La spiegazione è invece personalizzata, si avvale di esemplificazioni e di termini non necessariamente convenzionali, è centrata sul paziente: è volta cioè a rispondere alle esigenze soggettive dell'interlocutore tenendo conto del suo livello di istruzione, del suo desiderio di approfondimento, delle sue abilità cognitive, del suo stato emotivo. Anche quando si parla di "consenso informato" emerge chiaramente questo aspetto cruciale: in genere il linguaggio con cui sono redatti i moduli che il paziente dovrebbe leggere e sottoscrivere è tipicamente informativo e il più delle volte, quindi, poco comprensibile. II professionista che assuma un approccio autenticamente patientcentered dovrebbe puntare piuttosto a un "consenso spiegato", soffermandosi con il paziente sul contenuto del modulo e accertandosi della sua corretta interpretazione. È questo l'unico modo di verificare la consapevolezza del paziente riguardo alle caratteristiche e ai rischi di procedure e interventi per cui si richiede il suo consenso;

- promuovere l'empowerment del paziente. II concetto di empowerment si è sviluppato negli anni Settanta ed è attualmente applicato agli ambiti più diversi, dalla politica sociale alla riabilitazione. II termine può essere inteso sia come un processo sia come il suo risultato. Come processo di sviluppo e crescita personale esso porta gli individui ad essere protagonisti attivi delle proprie scelte e della propria vita, attraverso la conoscenza critica dell'ambiente e la percezione di poter esercitare un controllo su di esso. II potere cui si riferisce il processo di empowerment può essere inteso come la consapevolezza e l'aspettativa positiva di possedere le risorse necessarie per controllare e gestire in prima persona la realtà, contribuendo inoltre attivamente con idee, risorse e possibilità nuove. L'empowerment permette al paziente di usare autonomamente la propria intelligenza, esperienza, intuizione e creatività, di sviluppare il proprio controllo sulla 
situazione valutando realisticamente sia le risorse disponibili che i limiti presenti, attribuendo un significato agli eventi. Esso è favorito da un atteggiamento di corretto e realistico supporto e valorizzazione delle potenzialità del paziente, in termini di auto-efficacia, controllo della situazione, individuazione di significati, autodeterminazione;

- esplorare insieme al paziente le alternative di trattamento possibili e individuare - a fianco dei costi - i benefici di ciascuna, non soltanto in termini puramente organici, ma anche psicosociali, cercando di portare l'attenzione del paziente sulle possibili conseguenze o risvolti positivi della propria condizione;

- individuare insieme al paziente le possibilità di supporto sociale nella gestione della malattia, ove presenti;

- garantire al paziente la propria disponibilità a riconsiderare il percorso terapeutico in funzione di eventuali cambiamenti nella situazione oggettiva e soggettiva riportati dal paziente stesso.

Infine, occorre tener presente che l'operatore porta il proprio vissuto soggettivo nella relazione con il paziente. Purtroppo questo aspetto è troppo spesso scotomizzato, negato o addirittura ignorato. II professionista della salute tende ancora a vedere se stesso come osservatore esterno del comportamento del paziente, della sua malattia e del suo vissuto soggettivo.

Tuttavia, come ripetutamente ribadito dai grandi studiosi della fisica moderna, l'oggetto dell'osservazione scientifica è modificato dall'osservazione stessa, e quindi ciò che studiamo è l'effetto della nostra interazione con l'oggetto di studio. Questo effetto si amplifica quando l'oggetto di osservazione non è la natura inanimata, ma un essere vivente $\mathrm{e}$, in particolare, un nostro conspecifico, con il quale condividiamo meccanismi emotivi, cognitivi e motivazionali.

Qualsiasi interazione tra esseri umani non è mai unilaterale, ma chiama in gioco atteggiamenti, convinzioni, pregiudizi, valori e aspettative da entrambe le parti. Pochi studi hanno finora analizzato il vissuto dell'operatore nella relazione con il paziente: ciò evidenzia da un lato la scarsa attenzione a questo aspetto e dall'altro, forse, la tendenza degli stessi operatori ad evitare un argomento così direttamente connesso alla dimensione esperienziale e soggettiva della professione, quantomeno sul versante relazionale. È assolutamente necessario espandere gli studi in questo settore se si vuole avanzare verso un modello di relazione con il paziente in cui gli operatori siano formati e supportati a mettersi veramente in gioco come persone.

\section{CURA ED ETICA}

\subsection{Dimensioni personali}

Una credenza comune relativa alle professioni d'aiuto è che per poter svolgere al meglio il proprio lavoro l'operatore debba essere dotato di spirito di sacrificio, nonché porre al primo posto gli altri e il loro benessere. La scelta professionale, in realtà, viene compiuta non soltanto per aiutare il prossimo, ma anche come fonte di realizzazione personale. Gli individui passano gran parte della propria vita impegnati in attività lavorative per le quali hanno studiato, nelle quali hanno investito tempo e risorse e dalle quali si aspettano gratificazione e riconoscimenti. In linea con la definizione dell'OMS, il benessere lavorativo non è sinonimo di assenza di disagio, ma di stato completo di salute nelle sue componenti fisiche, psicologiche e sociali. Esso include la sicurezza sul lavoro, ma anche la soddisfazione personale legata all'autoefficacia, all'autonomia, all'autostima, tutte componenti che estendono la propria influenza anche nelle sfere extra-lavorative, contribuendo alla soddisfazione e alla qualità di vita generali. La soddisfazione lavorativa dell'operatore, inoltre, incide sulla soddisfazione del paziente in merito alla qualità delle 
cure e sulla sua salute in termini di aderenza ai trattamenti, risoluzione dei sintomi e adattamento alla malattia.

Tra i fattori più rilevanti e più frequentemente riconosciuti dagli operatori sanitari figura la relazione con il paziente, in linea con i presupposti stessi delle helping professions. In particolare, costituiscono importanti fonti di gratificazione la comunicazione chiara e il feedback sul lavoro dell'operatore. Peraltro, poiché l'obiettivo dell'operatore è curare il paziente allo scopo di guarirlo, in reparti in cui la malattia è incurabile o cronica si registrano elevati punteggi di esaurimento emotivo. In particolare, la morte del paziente, le richieste impossibili, le minacce e la scarsa aderenza alle prescrizioni sono motivo di logorio psicofisico per l'operatore.

I ricercatori interessati al benessere lavorativo hanno identificato il costrutto dell'impegno (job engagement). Esso è costituito da tre dimensioni: energia, coinvolgimento ed efficacia. In una prospettiva più allargata, è possibile considerarlo come un aspetto della soddisfazione lavorativa dell'operatore sanitario, insieme ai fattori relazionali (ad es. il rapporto con il paziente), situazionali (ad es. la gestione del reparto) e individuali (ad es. l' autonomia).

II costrutto dell'impegno lavorativo offre interessanti implicazioni per l'intervento. Promuovere l'impegno implica favorire il benessere personale. A tale scopo può essere utile incrementare le risorse lavorative attraverso la gestione partecipativa, il sostegno sociale e il team building e, allo stesso tempo, fornire strumenti necessari per affrontare le richieste lavorative elevate, come gli orari flessibili.

Per quanto riguarda gli aspetti relazionali del lavoro, sono stati individuati alcuni ostacoli alla loro promozione: oltre alla carenza di formazione specifica dei professionisti occorre considerare la scarsità del tempo a disposizione per un colloquio, la bassa priorità comunemente attribuita a questi aspetti della cura, la mancanza di consapevolezza di molti operatori riguardo al proprio stile relazionale, l'assenza di riscontri dall'esterno sulle modalità individuali di interazione. A volte si pensa di aver comunicato adeguatamente e chiaramente con il paziente, mentre il messaggio può non essere stato recepito correttamente a causa di omissioni di informazioni, eufemismi fuorvianti o linguaggio troppo tecnico; inoltre, dopo aver ricevuto una cattiva notizia il paziente può trovarsi in uno stato emotivo alterato (ansia, depressione, rabbia) e ciò pregiudica la sua possibilità di comprensione dei contenuti successivi del colloquio; infine, l'operatore deve far fronte non solo alle reazioni emotive del paziente, ma anche al proprio vissuto personale riguardo alla malattia e alla morte. II senso di inadeguatezza può portare il professionista a restringere la conversazione con il paziente ad aree "emotivamente sicure", ad esempio - come il più delle volte accade - agli aspetti puramente biomedici; a mantenersi intenzionalmente distaccato e non empatico "per il bene del paziente"; a convincersi che l'attenzione alla dimensione psicosociale non è di sua competenza.

Tra le risorse che possono essere coltivate dall'operatore occorre innanzitutto citare l'autoefficacia, cioè la competenza percepita dall'individuo in una data situazione. L'autoefficacia favorisce il benessere in quanto la fiducia nella proprie capacità e l'aspettativa di risultati positivi promuovono l'impegno, la perseveranza di fronte agli ostacoli, la soddisfazione personale nel raggiungimento degli obiettivi. L'individuo è quindi in grado di auto-regolare il proprio funzionamento personale, attraverso i meccanismi cognitivi delle aspettative e convinzioni, che gli permettono di impegnarsi nel perseguimento di obiettivi anche dilazionati nel tempo. Numerosi studi hanno analizzato le modalità di sviluppo dell'auto-efficacia nel corso della vita e le sue conseguenze sul comportamento, nonché le strategie per supportarla ed implementarla in vari ambiti e circostanze.

Su presupposti analoghi si basa la Teoria dell'Auto-Determinazione, che ha permesso di evidenziare il ruolo della motivazione intrinseca nella promozione del benessere. Per "motivazione intrinseca" si intende la spinta ad agire sulla base dell'interesse e del 
coinvolgimento in un'attività, e non in funzione di scopi esterni all'attività o di aspettative di ricompense materiali o sociali. Alla base della motivazione intrinseca stanno tre bisogni psicologici innati, che supportano il funzionamento ottimale e lo sviluppo dell'individuo: il bisogno di competenza, il bisogno di autonomia e il bisogno di relazionalità. In particolare, i bisogni di autonomia e competenza sono chiaramente connessi all'auto-regolazione del comportamento e allo sviluppo di capacità. Vari studi hanno dimostrato che un comportamento può essere caratterizzato da motivazione intrinseca solo quando l'individuo percepisce di acquisire delle competenze, di poter agire in autonomia e di muoversi in un ambiente sicuro e favorevole. Questo ha portato anche all'analisi dei fattori ambientali che promuovono la motivazione intrinseca: ad esempio, un contesto educativo in cui i tre bisogni fondamentali dei bambini siano supportati attivamente da familiari e insegnanti favorisce l'apprendimento e le prestazioni nello studio. Numerosi studi ne hanno dimostrato l'importanza anche in ambito lavorativo.

Infine, la hardiness (letteralmente "forza d'animo") si configura come una delle risorse che un operatore può mettere in gioco. Essa comprende tre caratteristiche psicologiche: l'impegno, il controllo, la percezione di sfide. Queste caratteristiche permettono di individuare aspetti costruttivi in situazioni altamente stressogene, promuovendo pertanto l'adattamento ed il benessere.

\subsection{Dimensioni organizzative}

II benessere degli operatori presenta ricadute a livello organizzativo. I professionisti che riportano assenza di malattia fisica e mentale e presenza di benessere fisico e mentale registrano i più elevati livelli di produttività lavorativa in termini di impegno, numero di giorni persi o a orario ridotto, rispetto a individui con stato di salute incompleta o con malessere. Essi, inoltre, incidono in misura nettamente minore sui costi sanitari, in termini di infortuni sul lavoro, ricoveri, visite e prescrizioni mediche. Tra gli infermieri è stata ad esempio registrata una relazione negativa tra ore di lavoro e soddisfazione e una relazione positiva tra scarso reddito, basso grado di sicurezza lavorativa e insoddisfazione.

Vi sono inoltre caratteristiche dell'organizzazione connesse alla soddisfazione lavorativa. Esse si riferiscono, in particolare, al tipo di reparto a cui è assegnato il personale e alla sua gestione. All'interno delle équipe di lavoro si sono rilevati i vantaggi derivanti da una specifica tipologia di leadership nel promuovere la soddisfazione lavorativa. Si tratta della cosiddetta "leadership trasformazionale": essa è caratterizzata dall'attenzione al singolo collaboratore e da valorizzazione e supporto di autodeterminazione, autoefficacia, e cooperazione in ciascun ambito dell'équipe.

Coloro che hanno una buona preparazione professionale riescono meglio a far fronte alle richieste dei pazienti e derivano un senso di competenza dall'investimento delle proprie capacità. Per le stesse ragioni, i programmi di educazione continua sembrano incidere positivamente sulla soddisfazione lavorativa. È possibile agire attraverso strategie mirate all'individuo, come programmi di educazione alla relazione, potenziamento dell'autoefficacia, del senso di coerenza; si possono utilizzare inoltre strategie centrate sull'organizzazione che incoraggino l'autonomia decisionale, offrano mansioni interessanti (e non noiose e ripetitive), favoriscano percezione di competenza ed esperienze complesse e gratificanti. In questo modo, è possibile promuovere la soddisfazione lavorativa e, conseguentemente, il benessere dell'operatore, dei pazienti e della struttura. I conflitti con colleghi, collaboratori e superiori sono fattori frequentemente connessi al burn-out degli operatori. Trattandosi di relazioni emotivamente rilevanti, in cui è in gioco il proprio status lavorativo e la propria autonomia, non sorprende la frequenza con cui i professionisti della salute li citano come problemi. In particolare, il supporto sociale rappresenta un'importante risorsa nelle professioni sanitarie: la mancanza di sostegno, specialmente da parte di supervisori o di colleghi, è legata al burn-out. 
II carico lavorativo si manifesta soprattutto come elevato numero di ore lavorative e di pazienti affidati, turni notturni (e ore di sonno limitate), carenza di personale.

Tra le caratteristiche dell'organizzazione, la struttura di lavoro o quella dell'équipe e i valori su cui tali strutture poggiano influenzano profondamente l'esperienza associata alle mansioni quotidiane. Altre caratteristiche problematiche della vita aziendale sono i periodi di ridimensionamento dell'organico, la flessibilità lavorativa e i passaggi di gestione.

Per favorire il benessere a lungo termine degli operatori deve mutare il concetto di lavoro: occorre passare da un'interpretazione weberiana di sofferenza e sacrificio ad una visione positiva che si focalizzi sulla realizzazione delle potenzialità umane e sui loro effetti crescita per la collettività (descritta più in dettaglio nel paragrafo successivo come visione eudaimonica). È questa la sfida che organizzazioni e lavoratori dovranno accogliere negli anni a venire.

\section{DALL'ESPERIENZA PERSONALE ALL'ESPERIENZA CONDIVISA}

Nella moderna concezione scientifica occidentale la valutazione del benessere si fonda su parametri oggettivi: le condizioni fisiche, il reddito, la situazione abitativa. Tuttavia, numerose ricerche hanno dimostrato che tali indicatori non forniscono un'immagine corretta delle risorse e delle potenzialità di sviluppo individuali. È altrettanto fondamentale valutare indicatori soggettivi del benessere e della qualità della vita, tenendo in considerazione la prospettiva e il vissuto del singolo individuo. È importante cioè chiedere direttamente alle persone quanto si sentano soddisfatte della propria vita, quali siano i loro obiettivi e come intendano perseguirli, quali siano le cose che provano piacere a fare e gli ambiti in cui si sentono più competenti e dotate.

Queste considerazioni hanno promosso l'attuale fiorire delle ricerche sulla percezione soggettiva del benessere e della qualità di vita. Gli studi in questo ambito derivano da due prospettive: quella edonica e quella eudaimonica. La prima identifica il benessere come soggettivo (SWB = subjective well-being) e lo riferisce principalmente alla dimensione affettiva (presenza di emozioni positive e assenza di emozioni negative) e a quella psicofisica, come ricerca del piacere e della soddisfazione. La seconda prospettiva, invece, definisce il benessere come psicologico (PWB = psychological well-being) e lo riferisce fondamentalmente all'auto-realizzazione, considerata come realizzazione delle potenzialità, risorse e predisposizioni individuali. Nella prospettiva eudaimonica (in linea con il significato originale attribuito da Aristotele al termine eudaimonia), il benessere non è necessariamente sinonimo di piacere: vengono piuttosto enfatizzate la capacità di perseguire obiettivi complessi e significativi per il singolo e la società, la mobilizzazione delle risorse in funzione di un aumento dell'autonomia e della condivisione, le competenze sociali e il ruolo delle relazioni interpersonali nella promozione del benessere.

Questa prospettiva presenta specifiche implicazioni per le possibili ricadute applicative. I dati emersi dagli ormai numerosi studi in questo ambito mostrano l'importanza di favorire il reperimento di esperienze positive e gratificanti in attività complesse e ricche di opportunità e sfide. Inoltre, gli stessi studi indicano l'estrema rilevanza, ai fini della promozione del benessere, del perseguimento di obiettivi significativi e utili allo sviluppo della complessità dell'individuo e della società in cui vive.

Programmi di intervento in campo educativo, preventivo, riabilitativo, organizzativo dovrebbero prestare attenzione alla complessità delle azioni individuali e delle opportunità ambientali. Ciò favorirebbe ad un tempo la crescita personale dei singoli, la loro integrazione sociale e lo sviluppo della comunità in cui essi vivono e alla quale contribuiscono con il loro agire quotidiano. L'approccio eudaimonico enfatizza in particolare la relazione tra benessere del singolo e sviluppo della collettività, 
svincolandosi dall'angusta visione individualistica che spesso caratterizza le ricerche psicologiche e mediche.

Studi sui valori culturali e interculturali hanno reso evidente la sostanziale tendenza di singoli e gruppi a livelli di complessità sempre più elevati. Se questo principio fondamentale non si manifesta nella realtà fattuale, ciò dipende da fattori molteplici, individuali ed ambientali, che è giusto esplorare e comprendere. Tuttavia, muoversi all'interno di una Weltanschauung che abbia come riferimento la cognizione di ciò che è possibile, desiderabile e significativo per il singolo e per la comunità permette di prestare attenzione a risorse, punti di forza, processi di crescita e strategie di implementazione delle abilità e capacità in una prospettiva ampia, che non scinda lo sviluppo individuale dall'empowerment sociale, il benessere del singolo dalla complessificazione della cultura e delle culture.

Le norme, le consuetudini e le credenze culturali, congiuntamente ai fattori sociali ed economici, contribuiscono a formulare e definire le opportunità di azione, sviluppo e integrazione di individui e gruppi all'interno di una comunità. Questi aspetti multidimensionali della cultura devono essere tenuti in considerazione al fine di identificare percorsi efficaci di promozione del benessere. In una autentica prospettiva eudaimonica, ciascun individuo deve essere considerato un agente attivo di cambiamento e sviluppo della comunità. Ciò vale per tutti i membri di una comunità, indipendente dall'età, dal genere e dalla collocazione sociale. È vero a maggior ragione per i cosiddetti gruppi svantaggiati: persone con disabilità, anziani, persone in condizioni di disagio psicosociale, immigrati, minoranze. Gli individui non sono, di per sé, strutturalmente svantaggiati: lo diventano in un ambiente sociale o culturale in cui la loro condizione comporti conseguenze svantaggiose, a causa di qualche discrepanza rispetto alle aspettative e alle regole sociali.

Ciascun individuo deve essere incoraggiato a seguire il proprio percorso di complessità e condivisione, a usare efficacemente i propri talenti e punti di forza, a coltivare attività che favoriscano esperienze stimolanti e gratificanti, a perseguire l'autodeterminazione attraverso l'esercizio della libertà e della responsabilità.

\section{BIBLIOGRAFIA}

1. Delle Fave A, Bassi M. Psicologia e salute. Torino: UTET, 2007.

2. Delle Fave A. (a cura di) La condivisione del benessere. Il contributo della psicologia positiva. Milano: FrancoAngeli, 2007.

3. Bassi M., Delle Fave A. L'esperienza della disabilità: selezione psicologica e promozione del benessere. Psicologia della Salute, 1, 31-48, 2007.

4. Bassi, M., Delle Fave, A. Psicologia positiva e promozione della salute: intervento in ambito sanitario. Psicologia della Salute, 2, 5-8, 2008.

5. Nosenzo M.A., Colombo F., Corsi F., Faini I., Bossi M., Sartani A., Cattaneo M.T., Trabucchi E., Tosca N., Filippi N., Delle Fave A. Neoplasia mammaria e mobilizzazione di risorse personali e relazionali. Psicologia della Salute, 2, 9-22, 2008.

6. Preziosa A., Riva G., Delle Fave A. L'esperienza soggettiva dell'obesità: implicazioni diagnostico-terapeutiche. Psicologia della Salute, 2, 39-58, 2008.

7. Bassi M., Coppa R., Delle Fave A. Studiare medicina per condividere benessere: una ricerca transculturale in Ciad e in Italia. Psicologia della Salute, 2, 93-107, 2008. 